\title{
Initial results for patient setup verification using transperineal ultrasound and cone beam CT in external beam radiation therapy of prostate cancer
}

\author{
Anne Richter*, Bülent Polat, Ingulf Lawrenz, Stefan Weick, Otto Sauer, Michael Flentje and Frederick Mantel
}

\begin{abstract}
Background: Evaluation of set up error detection by a transperineal ultrasound in comparison with a cone beam $\mathrm{CT}$ (CBCT) based system in external beam radiation therapy (EBRT) of prostate cancer.

Methods: Setup verification was performed with transperineal ultrasound (TPUS) and CBCT for 10 patients treated with EBRT for prostate cancer. In total, 150 ultrasound and CBCT scans were acquired in rapid succession and analyzed for setup errors. The deviation between setup errors of the two modalities was evaluated separately for each dimension.

Results: A moderate correlation in lateral, vertical and longitudinal direction was observed comparing the setup errors. Mean differences between TPUS and CBCT were $(-2.7 \pm 2.3) \mathrm{mm},(3.0 \pm 2.4) \mathrm{mm}$ and $(3.2 \pm 2.7) \mathrm{mm}$ in lateral, vertical and longitudinal direction, respectively. The mean Euclidean difference between TPUS and CBCT was $(6.0 \pm 3.1) \mathrm{mm}$. Differences up to $19.2 \mathrm{~mm}$ were observed between the two imaging modalities.

Discrepancies between TPUS and CBCT of at least $5 \mathrm{~mm}$ occurred in $58 \%$ of monitored treatment sessions.

Conclusion: Setup differences between TPUS and CBCT are $6 \mathrm{~mm}$ on average. Although the correlation of the setup errors determined by the two different image modalities is rather week, the combination of setup verification by CBCT and intrafraction motion monitoring by TPUS imaging can use the benefits of both imaging modalities.
\end{abstract}

Keywords: Prostate cancer, Transperineal ultrasound, IGRT, Setup verification, Cone beam CT

\section{Background}

During external beam radiation therapy (EBRT) of prostate cancer both interfractional and intrafractional movements of the prostate commonly occur. Changes in filling levels of bladder and rectum cause internal organ movements particularly in the anterior-posterior direction [1-3]. Image-guided radiotherapy (IGRT) provides the position of anatomical structures prior to treatment application. The most common technique of IGRT uses cone beam computed tomography (CBCT) based systems. CBCT based systems enable a reliable detection of interfractional target organ movements, however with the disadvantage of additional radiation exposure to the

\footnotetext{
* Correspondence: richter_a3@ukw.de

Department of Radiation Oncology, University of Wuerzburg,

Josef-Schneider-Str. 11, 97080 Wuerzburg, Germany
}

patient. Non-radiation based systems use ultrasound [4], optical surface detection [5], electromagnetic tracking [6] or MRI [7, 8]. Optical systems are suitable in situations where an external surface may act as a reliable surrogate for internal position or motion. This is not the case for localization of the prostate. Electromagnetic tracking makes use of transponders embedded in the tumor requiring a minimal invasive procedure for implantation. MR guided systems can visualize the soft tissue with an accuracy of 1-2 $\mathrm{mm}$ [9]. Limitation of MR based systems exist for patients with metallic implants and pacemakers. Ultrasound (US) imaging provides an alternative solution for treatment setup verification without an invasive procedure or additional imaging radiation dose. The technique of transabdominal US was evaluated by several groups [10-13] while 
the present study is focused on the transperineal setup of the US device. To date, no reports on transperineal US scanning for prostate localization are available. However, there is an ongoing controversial discussion on the precision of existing US systems [4].

The aim of this work was to validate detected set up errors using the transperineal ultrasound probe with Autoscan capability. The Clarity system was evaluated in terms of accuracy in patient set up control. The setup error values detected by the CBCT system served as reference values.

\section{Methods}

Setup verification was evaluated for ten patients who underwent EBRT for localized prostate cancer. The study was approved by the Ethics Committee of the University of Wuerzburg and all patients gave written informed consent. The patients were asked to reproduce their organ filling with an empty rectum and a filled bladder. The patients were advised to follow a drinking protocol and nutritional consultancy. The workflow of imaging for treatment planning and setup verification is illustrated in Fig. 1.

\section{Imaging and treatment planning}

For treatment planning, two image sets were acquired: a computed tomography (CT) scan (SOMATOM Sensation Open, Syngo CT, Siemens, Germany) and a 3D US reference scan (Clarity', Elekta, Stockholm, Sweden). Both scans were performed in rapid succession in the identical supine patient position, stabilized with kneefix, foot support and the transperineal ultrasound (TPUS) probe in place. Figure 2 shows the schematic patient setup with the TPUS. For target volume and organ at risk (OAR) delineation patients also had an MRI scan

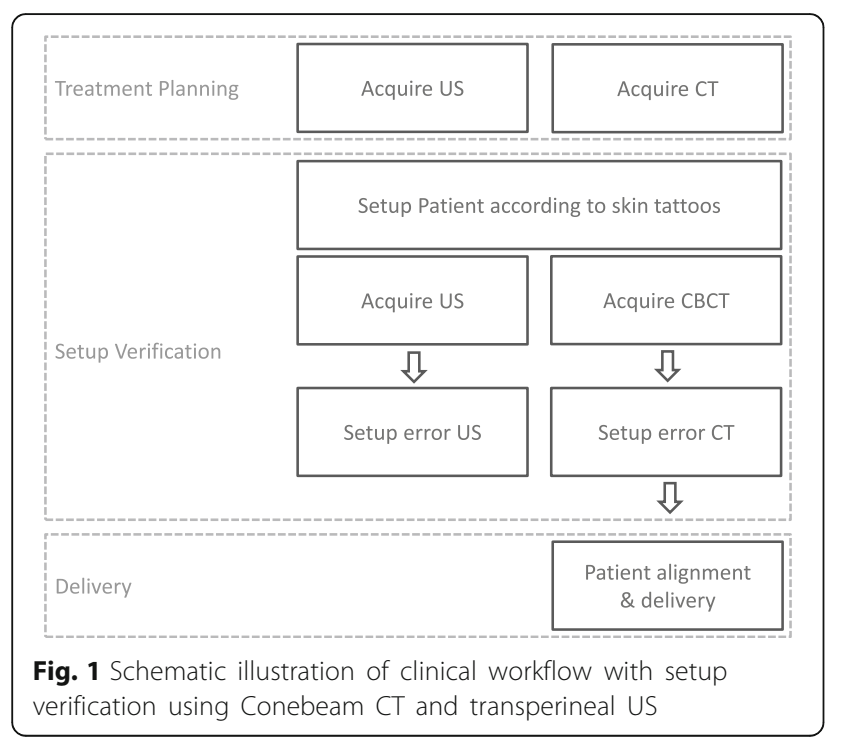

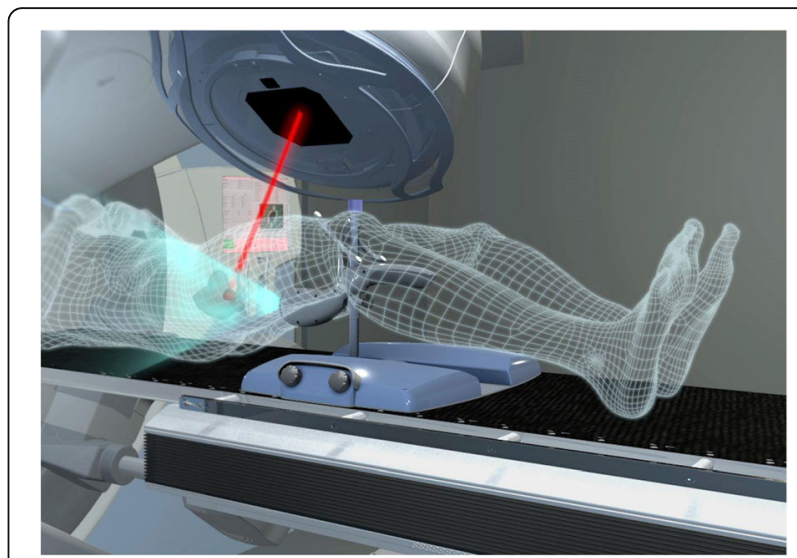

Fig. 2 Schematic patient setup for transperineal ultrasound

with a dummy US probe. The Clarity Autoscan system has integrated a mechanically-scanned probe as described by Lachaine et al. [14].

Treatment was planned for an Elekta Synergy Platform $^{\circ}$ equipped with Agility Head and XVI Conebeam system (Elekta Oncology Systems, Crawley, UK). Pinnacle (Philips Radiation Oncology Systems, Milpitas, CA, USA) was used for treatment planning of a volumetric modulated arc technique with a simultaneous integrated boost (SIB). Total doses were D95 of 60Gy to the PTV and mean dose of 76.2Gy to the boost volume given in 33 fractions. The protocol of target volume definition and treatment planning was described in detail before $[15,16]$.

CT images, contours and treatment plan, were transferred to the Clarity planning workstation and registered to the US reference scan. The prostate was delineated based on TPUS images and defined as the Positioning Reference Volume (PRV). The PRV served as reference structure for setup verification of the patient position via TPUS in the treatment room. Both, scan acquisition and PRV delineation were performed by trained general users.

\section{Imaging and setup verification}

Before treatment, the patient positioning was verified using the TPUS imaging directly followed by CBCT imaging. The setup error was recorded for both imaging systems for retrospective analysis. Compensation of the setup error and verification of treatment position was based on CBCT imaging. The planning $\mathrm{CT}$ and $\mathrm{CBCT}$ were registered automatically based on grey values followed by visual validation by the user. No rotational setup errors were determined, only translations were considered. CBCT imaging was performed daily for the first 7 fractions. Afterwards CBCT was acquired every third fraction. TPUS imaging was done for each fraction. The PRV was placed manually by the trained general 
user in the US image set. For each patient, setup verification with both image modalities was available in 15 fractions.

\section{Comparison of prostate localization}

First, setup errors were analyzed separately for each dimension to find out if there is any predominant direction with a large deviation. Evaluation in lateral, vertical and longitudinal direction corresponded to left-right, anterior-posterior and superior-inferior direction, respectively. The $3 \mathrm{D}$ vectors (Euclidean distance) of the setup errors were then compared. The overall mean was calculated from the absolute differences between CBCT and TPUS. The difference between CBCT and TPUS prostate localization was analyzed in a correlation and BlandAltman plot [17]. The limits of agreement (Mean $\pm 2 \%$ SD) indicate if the two methods are interchangeable and if the difference between the upper and lower limit is within a clinically acceptable range. Significance of the differences among TPUS imaging and CBCT was tested by the paired two-sided Student's $t$-test.

\section{Results}

In total 150 TPUS- and CBCT scans were acquired sequentially and analyzed to verify the treatment position. The obtained setup errors from the TPUS and CBCT imaging were compared. Results are listed in Table 1.

\section{Setup error distribution of TPUS and CBCT imaging}

For $\mathrm{CBCT}$ imaging, setup errors ranged from -7.3 to $12.1 \mathrm{~mm}$, from -12.0 to $7.7 \mathrm{~mm}$ and from -10.3 to
$9.7 \mathrm{~mm}$ in lateral, vertical and longitudinal direction, respectively. The absolute mean setup error for CBCT imaging was $(1.1 \pm 3.6) \mathrm{mm},(0.3 \pm 3.3) \mathrm{mm}$ and $(0.9 \pm 3.2)$ $\mathrm{mm}$ in lateral, vertical and longitudinal direction, respectively.

For US imaging, a larger setup error range was measured with ranges of -12.6 to $10.3 \mathrm{~mm},-12.6$ to $11.0 \mathrm{~mm}$ and -11.9 to $11.3 \mathrm{~mm}$ in lateral, vertical and longitudinal direction, respectively. The absolute mean setup errors for US imaging were $(-0.4 \pm 3.8) \mathrm{mm},(0.8 \pm 4.1) \mathrm{mm}$ and $(-0.5 \pm 4.4) \mathrm{mm}$ in lateral, vertical and longitudinal direction, respectively. In $70 \%$ and $49 \%$ of all TPUS and $\mathrm{CBCT}$ sessions the $3 \mathrm{D}$ setup error of $5 \mathrm{~mm}$ was exceeded.

\section{Comparison of TPUS and CBCT imaging}

The setup errors of TPUS imaging as a function of CBCT imaging are plotted in Fig. 3a-c for each dimension. There is only poor agreement of the data points with the line of equality. Pearson correlation coefficients of $-0.57,0.49$ and 0.49 were calculated in lateral, vertical and longitudinal direction, respectively. The strength of linear correlation was moderate in all direction. The correlation between CBCT and TPUS setup errors was significant in all direction $(p<0.01)$ only. By the twosided paired Student's $t$-test, there was no significant difference between TPUS and CBCT in vertical direction $(p=0.07)$ whereas there was significant difference in lateral and longitudinal direction $(p<0.001)$.

The difference between setup error measurements as a function of the mean of both values is shown in a BlandAltmann analysis in Fig. 3. The upper and lower limits of

Table 1 Summary of setup errors: mean, standard deviation, median and range

\begin{tabular}{|c|c|c|c|}
\hline Dimension & Mean \pm STD & Median & Range \\
\hline \multicolumn{4}{|l|}{ Lateral } \\
\hline US imaging & $-0.4 \pm 3.8$ & -0.3 & $-12.6 \rightarrow 10.3$ \\
\hline $\mathrm{CBCT}$ imaging & $1.1 \pm 3.6$ & 1.4 & $-7.3 \rightarrow 12.1$ \\
\hline Absolute difference of US and CBCT & $2.7 \pm 2.3$ & 2.5 & $0 \rightarrow 10.3$ \\
\hline \multicolumn{4}{|l|}{ Vertical } \\
\hline US imaging & $0.8 \pm 4.1$ & 1.0 & $-12.6 \rightarrow 11.0$ \\
\hline $\mathrm{CBCT}$ imaging & $0.3 \pm 3.3$ & 1.2 & $-12.0 \rightarrow 7.7$ \\
\hline Absolute difference of US and CBCT & $3.0 \pm 2.4$ & 2.5 & $0 \rightarrow 11.8$ \\
\hline \multicolumn{4}{|l|}{ Longitudinal } \\
\hline US imaging & $-0.5 \pm 4.4$ & -0.8 & $-11.9 \rightarrow 11.3$ \\
\hline $\mathrm{CBCT}$ imaging & $0.9 \pm 3.2$ & 0.6 & $-10.3 \rightarrow 9.7$ \\
\hline Absolute difference of US and CBCT & $3.2 \pm 2.7$ & 2.6 & $0 \rightarrow 16.5$ \\
\hline \multicolumn{4}{|l|}{$3 \mathrm{D}$} \\
\hline US imaging & $6.0 \pm 2.9$ & 6.3 & $0.8 \rightarrow 16.9$ \\
\hline CBCT imaging & $5.4 \pm 2.7$ & 4.9 & $0.6 \rightarrow 13.3$ \\
\hline Absolute difference of US and CBCT & $6.0 \pm 3.1$ & 5.6 & $1.1 \rightarrow 19.2$ \\
\hline
\end{tabular}

In units of $\mathrm{mm}$ 

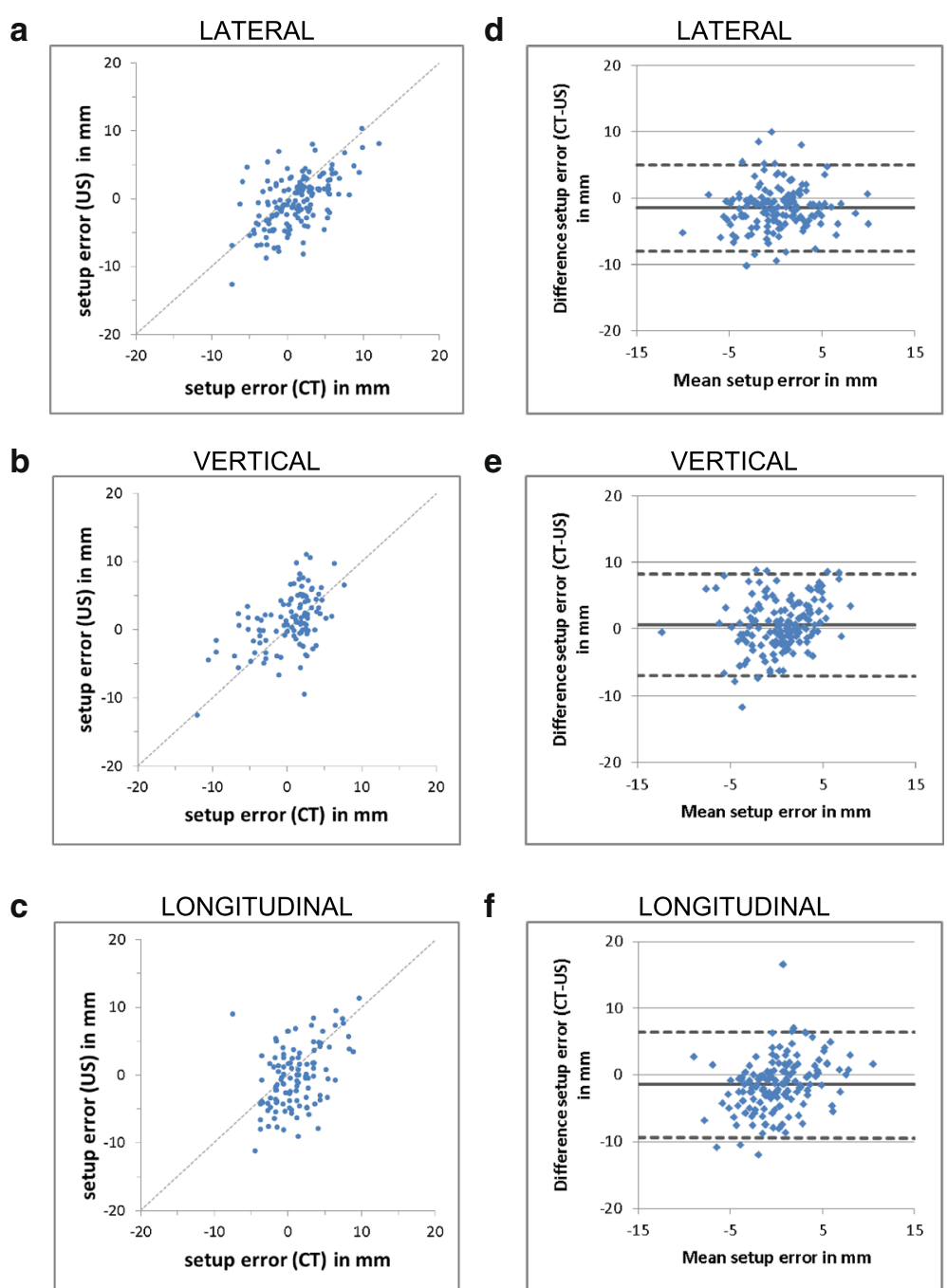

Fig. 3 Setup errors measured with US and CBCT imaging shown in correlation plots with line of equality (a, b, c) and Bland-Altmann plots $(\mathbf{d}, \mathbf{e}, \mathbf{f})$

agreement were $5 \mathrm{~mm} /-8 \mathrm{~mm}, 8.2 \mathrm{~mm} /-7.1 \mathrm{~mm}$ and $6.5 \mathrm{~mm} /-9.4 \mathrm{~mm}$ in lateral, vertical and longitudinal direction, respectively.

The distribution of setup errors is summarized in boxplots in Fig. 4 for each dimension and imaging modality. The average distance between CBCT and TPUS was $(2.7 \pm 2.3) \mathrm{mm},(3.0 \pm 2.4) \mathrm{mm}$ and $(3.2 \pm 2.7) \mathrm{mm}$ in lateral, vertical and longitudinal direction, respectively. The boxplot in Fig. 4c shows the distribution of absolute distance between $\mathrm{CBCT}$ and TPUS in lateral, vertical and longitudinal direction. Similar values were calculated for the median deviation with $2.5 \mathrm{~mm}, 2.5 \mathrm{~mm}$ and $2.6 \mathrm{~mm}$ in lateral, vertical and longitudinal direction, respectively. As shown in Fig. 4c, the largest discrepancies between TPUS and CBCT were observed in longitudinal dimension with a median deviation of $2.5 \mathrm{~mm}$ and a maximum deviation of $16.5 \mathrm{~mm}$. In lateral dimension, the lowest deviation between both imaging systems was observed with a median difference of $2.5 \mathrm{~mm}$ and a maximum deviation of $10.3 \mathrm{~mm}$. The minimum observed discrepancy between CT and TPUS setup errors in $3 \mathrm{D}$ was $1.1 \mathrm{~mm}$, while the maximum was $19.2 \mathrm{~mm}$. The resulting mean deviation in $3 \mathrm{D}$ was $(6.0 \pm 3.1) \mathrm{mm}$ with a median deviation of $5.6 \mathrm{~mm}$. The difference between TPUS and CBCT was larger than $5 \mathrm{~mm}$ in $58 \%$ of all monitored treatment sessions. Differences larger than $10 \mathrm{~mm}$ occurred in $11 \%$ of the monitored treatment sessions.

\section{Discussion}

Comparison of IGRT techniques

Mean differences between TPUS and CBCT determined shifts of 2 to $3 \mathrm{~mm}$ for each dimension were observed. The resulting Euclidean distance was $6 \mathrm{~mm}$ on average. 

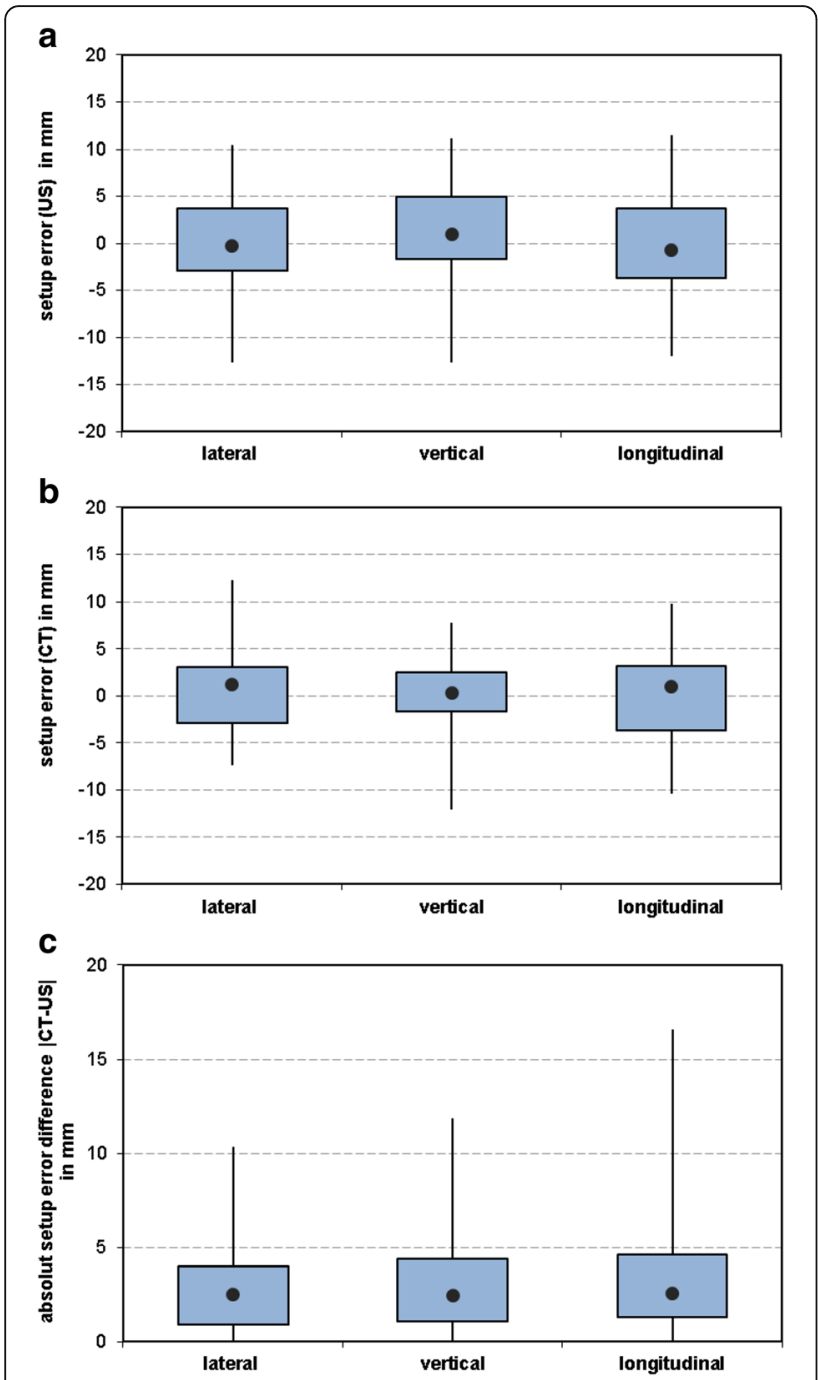

Fig. 4 Boxplots of setup errors for (a) US, (b) CBCT imaging and (c) CT-US in lateral, vertical and longitudinal dimension

However, for individual setups differences up to $20 \mathrm{~mm}$ occurred. The difference between TPUS and CBCT was larger than $5 \mathrm{~mm}$ and $10 \mathrm{~mm}$ in $58 \%$ and $11 \%$ of all monitored treatment sessions.

Robinson et al. evaluated the differences between $\mathrm{CBCT}$ and transabdominal US imaging. They reported a mean discrepancy of $10 \mathrm{~mm}$ while our evaluation resulted in an average distance of $6 \mathrm{~mm}$ [12]. Other studies compared the accuracy of setup verification for implanted fiducial markers and US in each cartesian direction $[11,13]$. The mean differences in setup errors were up to $2-3 \mathrm{~mm}$ which is comparable to our results with a median deviation of $2.5-2.6 \mathrm{~mm}$ deviation.

In our study, larger differences were observed in longitudinal direction $(3.2 \pm 2.7) \mathrm{mm}$ while smaller deviations were seen in lateral direction $(2.7 \pm 2.3) \mathrm{mm}$. This is in agreement to the results published by other groups
$[11,13]$. The Bland-Altmann analysis resulted in limits of agreement of 8 to $10 \mathrm{~mm}$ covering $95 \%$ of the measurements. The largest limit of agreement was calculated in longitudinal direction $(6.5 \mathrm{~mm} /-9.4 \mathrm{~mm})$. This is in agreement to the results published by van der Meer et al. who obtained a limit of agreement of $10 \mathrm{~mm}$ in longitudinal direction [13]. In contrast, Robinson et al. observed the greatest disparity between $\mathrm{CT}$ and transabdominal US in the vertical direction [12].

Discrepancies between TPUS and CBCT of $5 \mathrm{~mm}$ and more occurred in $58 \%$ of our monitored treatment sessions. Discrepancies of $10 \mathrm{~mm}$ were exceeded in $11 \%$. Again, Robinson et al. observed larger deviations: $5 \mathrm{~mm}$ were exceeded in $89 \%$ and $10 \mathrm{~mm}$ were exceeded in $42 \%$ of the measurements [12]. Van der Meer et al. showed that in $56 \%$ of all patients the difference in at least one direction was larger than $5 \mathrm{~mm}(>10 \mathrm{~mm}$ for $11 \%)$ [13]. In the present study, the distribution of setup errors showed a larger range for TPUS $(0.8-16.9 \mathrm{~mm})$ than for CBCT $(0.6-13.3 \mathrm{~mm})$. The mean and standard deviation were slightly smaller for $\mathrm{CBCT}$ imaging (TPUS: $6.0 \pm 2.9 \mathrm{~mm}$ and CBCT: $5.4 \pm 2.7 \mathrm{~mm}$ ). The absolute difference in $3 \mathrm{D}$ between both imaging modalities was $(6.0 \pm 3.1) \mathrm{mm}$ with a median deviation of $5.6 \mathrm{~mm}$. Subsequently, setup differences between TPUS and $\mathrm{CBCT}$ are $6 \mathrm{~mm}$ on average. These preliminary results will be examined in a larger patient population in our further research. Some groups report that the accuracy of US is comparable to fiducial marker based localization $[11,13]$ while another group concluded that the US fails to deliver an acceptable level of geometric accuracy with regard to prostate localization [12].

In comparison to US, external localization systems like MRI-guided IGRT and electromagnetic tracking systems achieve a geometric accuracy of 1-2 $\mathrm{mm}[9,18]$. Mayyas et al. compared the interfraction setup errors between different localization techniques $(\mathrm{kV}, \mathrm{CBCT}$ and US imaging and electromagnet transponders) [19]. They found that that the $\mathrm{kV}$ and $\mathrm{CBCT}$ shifts are comparable to that of US.

Image acquisition for $\mathrm{CBCT}$ is a user independent technique, which is widely used for setup verification during radiation therapy for prostate cancer [20]. However, CBCT leads to additional patient dose. That is admittedly low in comparison to the treatment related dose, but is not fully negligible in the patient cohort of prostate EBRT with a relatively favorable prognosis. The $\mathrm{CBCT}$ is reasonably not used for setup verification in every treatment session. Whereas, a daily use of the US imaging is very well possible and can potentially detect a disadvantageous bladder and rectum filling earlier. This information can be incorporated in the treatment workflow by asking the patient to adapt bladder or rectum filling. 
Besides setup verification, the transperineal setup of the US device additionally offers the possibility of intrafraction target monitoring and possible motion management in the future. A first proof of principle experiment of US based motion tracking was performed in a phantom study by Schwaab et al. with promising results but the need of further research [21]. Colvill et al. studied the dosimetric impact of tracking and gating to account for intrafraction motion during prostate radiotherapy [22]. O'Shea et al. reviewed the applicability of intrafraction monitoring with US for prostate and other treatment sites [23]. Until further results on the practicability and accuracy of TPUS are available, certainly the combination of setup verification by $\mathrm{CBCT}$ and intrafraction target organ and OAR motion monitoring by TPUS imaging can use the benefits of both imaging modalities.

\section{Limitations}

One limitation is that no rotational errors were determined for prostate positioning. The US imaging and registration offers translational errors only. For setup verification of patients with prostate cancer, we correct for translational errors according to our protocol due to practicability and patient stability. Effects of rotations and translations of the prostate should be taken into account during target delineation and margin definition. The correction of rotational errors becomes more important for the hypofractionated radiotherapy treatment of prostate cancer and treatment of large, complex target volumes or simultaneous, single isocenter irradiation of multiple targets [24]. The correction of rotational errors can induce substantial secondary patient displacements if the patient is not immobilized sufficiently [25].

Another limitation of our study is the small number of patients. The results will be examined in a larger patient population in further research.

The applicability of US for prostate localization is discussed controversially in the literature due to its uncertainties like user variability (scanning variability and registration variability). Different probe pressures during image acquisition can lead to unreproducible target organ movements $[4,11-13]$. The influence of the probe pressure on the reproducibility is still an issue of concern $[4,10,13,16]$. The advantage of the Clarity system is the Autoscan probe decreasing the scanning variability. As we found out in a recently published study, the transperineal probe setup shifted the penile bulb in cranial direction and thereby increased the dose to the penile bulb [16]. However, in that study, no relevant changes in the prostate or PTV volumes were observed and organ motion of the prostate was only seen to a minor extent mainly in the superior direction. [26]. This seems to substantiate the assumption that probe pressure induced organ motion is reduced to a minimum by the transperineal scanning approach compared to transabdominal US. The variability of probe pressure is further reduced within the TPUS Clarity ${ }^{\circ}$ system by probe fixation and a height scale as well as the display of the current and planned probe position in the software. Nonetheless, as low pressure of the TPUS probe as feasible to still achieve appropriate image quality is recommended for limitation of structure shifts and further reduction of placement variability.

User variability is influenced not only by scanning, but also by image registration and the manual placement of the Reference Positioning Volume. For the transabdominal US approach, van der Meer et al. reported a user variability of $2-3 \mathrm{~mm}$ - including scanning and matching variability [13]. Van der Meer determined the intraand interoperator variability for transabdominal US. In their study, the intraoperator match variability was in the range of $1 \mathrm{~mm}$ and the interoperator variability was in the range of $1.3-1.8 \mathrm{~mm}$. Because the accuracy of US is highly influenced by the user more effort is required to standardize US imaging compared to CBCT or portal imaging, as van der Meer already stated [13]. This certainly includes a comprehensive employee training but also improvements from the technical side are warranted like automated image registration and segmentation.

\section{Conclusion}

The evaluation of setup verification with TPUS and CBCT showed setup differences of $6 \mathrm{~mm}$ on average. Due to its large user dependency more effort is required to standardize US imaging compared to CBCT or portal imaging. At the moment, the combination of setup verification by $\mathrm{CBCT}$ and intrafraction motion monitoring by TPUS imaging can use the benefits of both imaging modalities.

\section{Acknowledgements}

Not applicable.

\section{Funding}

This research was partially supported through a research grant from Elekta $A B$, Sweden. Elekta had no involvement in study design, data collection and analysis. This publication was supported by the Open Access Publication Fund of the University of Wuerzburg.

Availability of data and materials Not applicable.

Authors' contributions

FM, IL, MF and BP designed the study. AR, FM, IL, MF, OS and SW participated in the data collection and analysis. All authors performed critical review of the manuscript and finally approved the manuscript.

Competing interests

All authors declare that they have no competing interests.

Consent for publication

Not applicable. 


\section{Ethics approval and consent to participate}

This study was reviewed and approved by the local institutional ethics committee (reference number 281/14).

Received: 12 July 2016 Accepted: 27 October 2016

Published online: 08 November 2016

\section{References}

1. Mah D, Freedman G, Milestone B, Hanlon A, Palacio E, Richardson T, Movsas B, Mitra R, Horwitz E, Hanks GE. Measurement of intrafractional prostate motion using magnetic resonance imaging. Int J Radiat Oncol Biol Phys. 2002;54(2):568-75.

2. Kupelian PA, Langen KM, Willoughby TR, Zeidan OA, Meeks SL. Imageguided radiotherapy for localized prostate cancer: treating a moving target. Semin Radiat Oncol. 2008;18(1):58-66

3. Ghilezan MJ, Jaffray DA, Siewerdsen JH, Van Herk M, Shetty A, Sharpe MB, Zafar Jafri S, Vicini FA, Matter RC, Brabbins DS, et al. Prostate gland motion assessed with cine-magnetic resonance imaging (cine-MRI). Int J Radiat Oncol Biol Phys. 2005;62(2):406-17.

4. Fontanarosa D, van der Meer S, Bamber J, Harris E, O'Shea T, Verhaegen F. Review of ultrasound image guidance in external beam radiotherapy: I. Treatment planning and inter-fraction motion management. Phys Med Biol. 2015;60(3):R77-R114

5. Brahme A, Nyman P, Skatt B. 4D laser camera for accurate patient positioning, collision avoidance, image fusion and adaptive approaches during diagnostic and therapeutic procedures. Med Phys. 2008;35(5):1670-81.

6. Foster RD, Solberg TD, Li HS, Kerkhoff A, Enke CA, Willoughby TR, Kupelian PA. Comparison of transabdominal ultrasound and electromagnetic transponders for prostate localization. J Appl Clin Med Phys. 2010;11(1):2924

7. Fallone BG, Murray B, Rathee S, Stanescu T, Steciw S, Vidakovic S, Blosser E, Tymofichuk D. First MR images obtained during megavoltage photon irradiation from a prototype integrated linac-MR system. Med Phys. 2009;36(6):2084-8

8. Raaymakers BW, Lagendijk JJ, Overweg J, Kok JG, Raaijmakers AJ, Kerkhof EM, van der Put RW, Meijsing I, Crijns SP, Benedosso F, et al. Integrating a 1. 5 T MRI scanner with a 6 MV accelerator: proof of concept. Phys Med Biol. 2009;54(12):N229-37.

9. Goyal S, Kataria T. Image guidance in radiation therapy: techniques and applications. Radiol Res Pract. 2014;2014:705604.

10. Dobler B, Mai S, Ross C, Wolff D, Wertz H, Lohr F, Wenz F. Evaluation of possible prostate displacement induced by pressure applied during transabdominal ultrasound image acquisition. Strahlentherapie und Onkologie: Organ der Deutschen Rontgengesellschaft [et al]. 2006;182(4):240-6.

11. Li M, Ballhausen H, Hegemann NS, Ganswindt U, Manapov F, Tritschler S, Roosen A, Gratzke C, Reiner M, Belka C. A comparative assessment of prostate positioning guided by three-dimensional ultrasound and cone beam CT. Radiat Oncol. 2015:10:82

12. Robinson D, Liu D, Steciw S, Field C, Daly H, Saibishkumar EP, Fallone G, Parliament M, Amanie J. An evaluation of the Clarity 3D ultrasound system for prostate localization. J Appl Clin Med Phys. 2012;13(4):3753.

13. van der Meer S, Bloemen-van Gurp E, Hermans J, Voncken R, Heuvelmans D, Gubbels C, Fontanarosa D, Visser P, Lutgens L, van Gils F, et al. Critical assessment of intramodality 3D ultrasound imaging for prostate IGRT compared to fiducial markers. Med Phys. 2013;40(7):071707.

14. Lachaine M, Falco T. Intrafractional prostate motion management with the Clarity Autoscan system. Medical physics international. 2013;1(1):1972-80.

15. Guckenberger M, Ok S, Polat B, Sweeney RA, Flentje M. Toxicity after intensity-modulated, image-guided radiotherapy for prostate cancer Strahlentherapie und Onkologie : Organ der Deutschen Rontgengesellschaft [et al]. 2010;186(10):535-43.

16. Mantel F, Richter A, Groh C, Lawrenz I, Weick S, Polat B, Guckenberger M, Flentje M. Changes in penile bulb dose when using the Clarity transperineal ultrasound probe: A planning study. Pract Radiat Oncol. 2016;6(6):e337-e344.

17. Bland JM, Altman DG. Statistical methods for assessing agreement between two methods of clinical measurement. Lancet. 1986;1(8476):307-10.

18. Willoughby TR, Kupelian PA, Pouliot J, Shinohara K, Aubin M, Roach 3rd M, Skrumeda LL, Balter JM, Litzenberg DW, Hadley SW, et al. Target localization and real-time tracking using the Calypso 4D localization system in patients with localized prostate cancer. Int J Radiat Oncol Biol Phys. 2006;65(2):528-34.
19. Mayyas E, Chetty IJ, Chetvertkov M, Wen N, Neicu T, Nurushev T, Ren L, Lu M, Stricker H, Pradhan D, et al. Evaluation of multiple image-based modalities for image-guided radiation therapy (IGRT) of prostate carcinoma: a prospective study. Med Phys. 2013:40(4):041707.

20. Nabavizadeh N, Elliott DA, Chen Y, Kusano AS, Mitin T, Thomas Jr CR, Holland JM. Image Guided Radiation Therapy (IGRT) Practice Patterns and GRT's Impact on Workflow and Treatment Planning: Results From a National Survey of American Society for Radiation Oncology Members. Int J Radiat Oncol Biol Phys. 2016;94(4):850-7.

21. Schwaab J, Prall M, Sarti C, Kaderka R, Bert C, Kurz C, Parodi K, Gunther M, Jenne J. Ultrasound tracking for intra-fractional motion compensation in radiation therapy. Phys Med. 2014;30(5):578-82.

22. Colvill E, Poulsen PR, Booth JT, O'Brien RT, Ng JA, Keall PJ. DMLC tracking and gating can improve dose coverage for prostate VMAT. Med Phys. 2014;41(9):091705.

23. O'Shea T, Bamber J, Fontanarosa D, van der Meer S, Verhaegen F, Harris E. Review of ultrasound image guidance in external beam radiotherapy part II: intra-fraction motion management and novel applications. Phys Med Biol. 2016;61(8):R90-R137.

24. Boda-Heggemann J, Lohr F, Wenz F, Flentje M, Guckenberger M. kV conebeam CT-based IGRT: a clinical review. Strahlentherapie und Onkologie : Organ der Deutschen Rontgengesellschaft [et al]. 2011:187(5):284-91.

25. Guckenberger M, Meyer J, Wilbert J, Baier K, Sauer O, Flentje M. Precision of image-guided radiotherapy (IGRT) in six degrees of freedom and limitations in clinical practice. Strahlentherapie und Onkologie : Organ der Deutschen Rontgengesellschaft [et al]. 2007:183(6):307-13.

26. Roach 3rd M, Nam J, Gagliardi G, El Naqa I, Deasy JO, Marks LB. Radiation dose-volume effects and the penile bulb. Int J Radiat Oncol Biol Phys. 2010;76(3 Suppl):S130-4.

\section{Submit your next manuscript to BioMed Central and we will help you at every step:}

- We accept pre-submission inquiries

- Our selector tool helps you to find the most relevant journal

- We provide round the clock customer support

- Convenient online submission

- Thorough peer review

- Inclusion in PubMed and all major indexing services

- Maximum visibility for your research

Submit your manuscript at www.biomedcentral.com/submit
) Biomed Central 